\title{
Fast and Slow Time Regimes of Fluorescence Quenching in Conjugated Polyfluorene-Fluorenone Random Copolymers: The Role of Exciton Hopping and Dexter Transfer along the Polymer Backbone
}

\author{
Fernando B. Dias,* Matti Knaapila, and Andrew P. Monkman \\ OEM Research Group, Department of Physics, University of Durham, Durham DH1 3LE, UK
}

Hugh D. Burrows

Departamento de Química, Universidade de Coimbra, 3004-535 Coimbra, Portugal

Received November 23, 2005; Revised Manuscript Received January 4, 2006

\begin{abstract}
The luminescence decay kinetics of polyfluorene copolymers containing fluorenone units randomly distributed along the polymer chain have been studied by steady-state and time-resolved fluorescence techniques in toluene solution. The typical green emission from polyfluorenes containing 9-fluorenone moieties is only observed if the 9-fluorenone group is covalently attached to the polymer. Small-angle neutron scattering (SANS) measurements indicate that, independent of the 9-fluorenone fraction, all the studied copolymers adopt an open wormlike conformation. This prevalent 1-dimensional arrangement confirms that the green emission observed with polyfluorenes is not the result of excimer formation in the typical sandwich-like conformation. Analysis of time-resolved fluorescence decays by the maximum entropy method (MEM) collected at the polyfluorene emission $(415 \mathrm{~nm})$ and by global analysis of decays collected at 415 and $580 \mathrm{~nm}$ (the 9-fluorenone defect emission wavelength) clearly indicates two different time regimes in the population of the fluorenone defect: one occurring in the time interval of 10 to $30 \mathrm{ps}$ and a second one occurring in the time range from 70 to $200 \mathrm{ps}$. While the slower process shows a linear dependence with the 9-fluorenone fraction, compatible with a hopping migration process along the polymer chain, the faster process does not show such a dependence and instead suggests a short-range Dexter mechanism. These findings are in agreement with our previous work where the presence of a faster component was suggested.
\end{abstract}

\section{Introduction}

The dynamics of intrachain and interchain excitation energy migration in conjugated organic polymers has been the subject of many studies in recent years. ${ }^{1-9}$ The understanding of this process is crucial for the exploitation of conjugated polymers for possible applications in areas such as electroluminescent devices, ${ }^{10}$ solar cells ${ }^{11}$ and other photon-harvesting systems, ${ }^{9}$ and fluorescence sensing. ${ }^{12}$ Even from the fundamental point of view, the understanding of energy transfer in polymers is a key requirement to reveal the complex photophysical behavior observed in these systems. ${ }^{9,13}$ In this work we present the study of fluorescence quenching in copolymers containing 9,9dialkylfluorene and 9-fluorenone units in order to bring a deeper insight into the role of energy migration along the polymer backbone on the population of oxidative defects in fluorenebased materials.

Resonant energy transfer (RET) describes the phenomenon of nonradiative energy transfer between two fluorophores with overlapping, emission (donor) and absorption (acceptor) spectra, resulting from the coupling between the donor fluorescence and acceptor excitation transitions. ${ }^{14}$

Conjugated polymers can be considered as an array of weakly coupled fluorophores with slightly different excitation energies. ${ }^{15}$ Disorder leads to a distribution of segments with different lengths and degrees of conjugation and, thus, with different $\pi-\pi^{*}$ transition energies. Thus, the absorption spectrum, in general, is strongly affected by inhomogeneous broadening at room temperature. ${ }^{13,16}$ Shorter segments have higher $\pi-\pi^{*}$

* To whom correspondence should be addressed. E-mail: f.m.b.dias@ durham.ac.uk. transition energies than longer ones and act as energy donors in RET toward the low-energy levels of the density of states (DOS). RET is in this way coupled to the distribution of conjugation lengths and strongly affected by fast fluctuations due to conformational reorganization of the polymer backbone. ${ }^{9,13,16-19}$

The effect of the polymer conformation on excitation dynamics is particularly relevant in dilute solutions. For polymers labeled with short lifetime chromophores, intrachain energy transfer steps along the polymer backbone of a single chain will predominate in the case of "rigid-rod" or open wormlike conformations. However, in a closed coil conformation, throughspace energy transfer between closely spaced segments of the same chain brought together as a result of chain folding ${ }^{1,9}$ could also occur, in direct analogue to interchain migration transfer.

We recently reported ${ }^{20}$ the study of intramolecular fluorescence quenching in (9,9-dialkylfluorene-co-fluorenone) copolymers $\left(\mathrm{PF} / \mathrm{FL}_{x}\right)$, with fluorenone fractions varying from $0.1 \%$ to $25 \%$. A kinetic mechanism involving three different kinetic species was proposed to explain the observations: (i) quenched polyfluorene excitations, (ii) unquenched polyfluorene excitations, and (iii) defects with charge transfer nature. The quenching rate constant was determined to be $0.12 \mathrm{ps}^{-1}\left(1.2 \times 10^{-11} \mathrm{~s}^{-1}\right)$. Strong evidence for the presence of an ultrafast decay component at higher fluorenone fractions was found but proved impossible to resolve due to experimental limitations.

In the present study, we add improved time resolution to clarify this point and to fully characterize the singlet excitedstate relaxation in these "photon-harvesting polymer" models. Of particular interest in the present report is the relation between the excited-state dynamics and the local molecular conformation. 


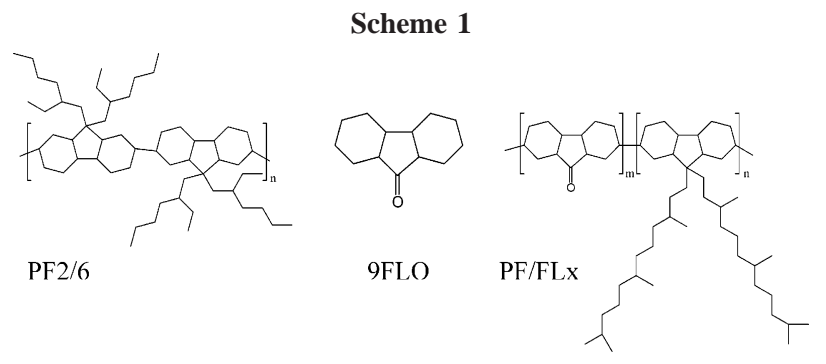

For example, how does energy relaxation occur? Is the assumption of quasi-one-dimensional geometry reasonable for these systems? Is the "intermolecular" energy transfer channel operative? Is it plausible to identify different species in the luminescence kinetics?

The intermolecular quenching of the homopolymer, poly[9,9di(ethylhexyl)fluorene] (PF2/6), emission by 9-fluorenone (9FLO) is found to occur only by the trivial reabsorption effect. Smallangle neutron scattering (SANS) measurements suggest prevalent one-dimensional wormlike conformation for all the copolymers studied. Both findings are in agreement with the assumption of intramolecular energy migration within a quasione-dimensional geometry, where the polymer acts as an antenna driving the excitation through subsequent segments of monomer units to the charge transfer defect that is acting as an energy trap.

\section{Experimental Section}

Synthesis and characterization of PF2/6 and of a series of poly[9,9-di(3,7,11-trymethyldodecyl)fluorene] copolymers with randomly distributed fluorenone fractions ranging from $0.1 \%$ to $25 \%$ $\left(\mathrm{PF} / \mathrm{FL}_{x}\right)$ have been described elsewhere, ${ }^{22} \overline{M_{\mathrm{w}}} \cong 10^{4}, \overline{M_{\mathrm{w}}} / \overline{M_{\mathrm{n}}} \cong$ 2.0. 9-Fluorenone (9FLO) was purchased from Aldrich and used after purification. See Scheme 1 for the structures of PF/FL ${ }_{x}, \mathrm{PF} 2 /$ 6 , and 9FLO.

Dilute solutions (concentration range $\sim 10^{-3} \mathrm{mg} / \mathrm{mL}, \mathrm{OD}<0.2$ ) of PF2/6 and PF/FL $x$ were prepared in toluene (purchased from ROMIL and used as supplied). Solutions of 9-fluorenone, used to study the intermolecular interaction between PF2/6 and 9FLO, were prepared using a toluene solution of PF2/6 $\left(10^{-3} \mathrm{mg} / \mathrm{mL}\right)$.

Absorption spectra were collected using a Perkin-Elmer LAMBDA 19 double-beam spectrophotometer in $1 \mathrm{~cm}$ path length quartz cells. Steady-state photoluminescence spectra of dilute solutions were collected on a fluorescence spectrometer (Fluorolog, Jobin Yvon) equipped with a xenon lamp as excitation source.

Time-resolved fluorescence decays were collected using the picosecond time correlated single photon counting technique (IRF $=25 \mathrm{ps}$ ). The excitation source, which has a vertical polarization, is a picosecond Ti:sapphire laser from Coherent Inc. (wavelength range $720-1000 \mathrm{~nm}, 76 \mathrm{MHz}$ repetition rate), coupled to a secondharmonic generator $(360-500 \mathrm{~nm})$. Emission collected at magic angle polarizer is detected through a double subtractive monochromator by a Hamamatsu microchannel plate (MCPT, model R3809U50). Signal acquisition was performed using a TCSPC module from Becker \& Hickl (model SPC-630). Deconvolution of the fluorescence decays was performed using an updated Linux version of the George Striker program ${ }^{23}$ and the Globals WE software package. ${ }^{24}$ The decays were repeated at least four times, and the average value of the decay times and amplitudes were then used in posterior data analysis. Maximum entropy method ${ }^{25}$ (MEM) analyses were made for decays collected at $415 \mathrm{~nm}$ (polyfluorene emission), with more than $5 \times 10^{4}$ counts accumulated in the channel of maximum counts, using a noncommercial software package. All measurements were done using degassed solutions.

Solutions of $\mathrm{PF} / \mathrm{FL}_{x}$ in deuterated toluene (D, 99.5\%) from Cambridge Isotope Laboratories Inc. $(10 \mathrm{mg} / \mathrm{mL})$ were used for SANS measurements at the LOQ beamline (ISIS facility), Rutherford Appleton Laboratory, UK. ${ }^{26}$ The LOQ instrument uses
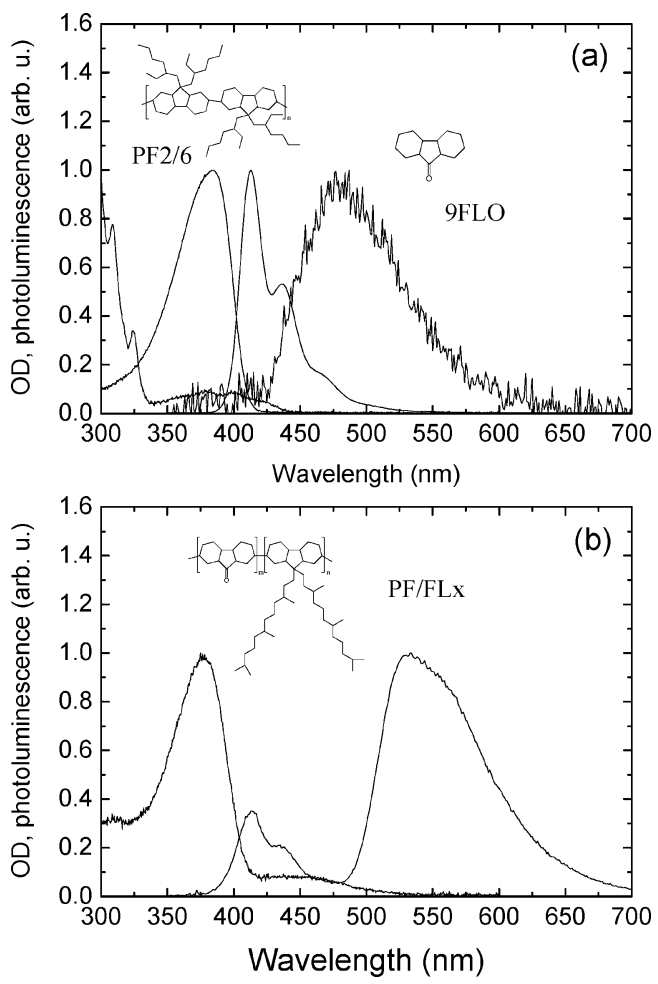

Figure 1. Normalized absorption and emission spectra of (a) PF2/6 and 9-fluorenone and (b) $\mathrm{PF} / \mathrm{FL}_{0.25}$ in toluene solution, $\lambda_{x}=390 \mathrm{~nm}$. The insets show the chemical structures of the compounds.

incident wavelengths between 2.2 and $10 \AA$ sorted by time-of-flight with a sample-to-detector distance of $4.1 \mathrm{~m}$, resulting in a $q$ range between 0.006 and $0.24 \AA^{-1}$. The samples were placed in $2 \mathrm{~mm}$ path length quartz cells (Hellma) kept in a thermostat at $25.0 \pm$ $0.5{ }^{\circ} \mathrm{C}$. The raw data were corrected for the transmission, background, sample cell, and detector efficiency. The incoherent scattering was ignored. The 2D scattering patterns were azimuthally averaged and converted to an absolute scale. The data for individual samples were collected for $30 \mathrm{~min}$.

\section{Results and Discussion}

A. Steady-State Absorption and Fluorescence Spectra. Figure 1 shows the absorption and emission spectra in toluene solution of (a) the homopolymer, PF2/6, and 9-fluorenone and (b) the copolymer PF/FL 0.25 randomly labeled with $25 \%$ of fluorenone units; the insets show the structures of the two polymers.

The absorption spectra of both polymers are characterized by a broad band between 300 and $425 \mathrm{~nm}$, which is the typical spectral signature of polyfluorene derivatives. However, in the case of $\mathrm{PF} / \mathrm{FL}_{0.25}$, an additional red-shifted band, attributed to a charge transfer state $8,20,21,27$ (CTS), is observed up to $525 \mathrm{~nm}$.

Spectral changes are also clearly observable in the emission spectra. For PF2/6, the typical narrow and well-resolved spectrum is observed, independent of the excitation wavelength. However, for PF/ $\mathrm{FL}_{0.25}$, besides the typical polyfluorene emission, excitation along the polyfluorene absorption band leads to an additional featureless emission, centered at $530 \mathrm{~nm}$; this emission results from the "CTS-defect" population at the expense of the polyfluorene excited state. ${ }^{20}$ Direct excitation of the red-shifted absorption band $(450 \mathrm{~nm})$, only gives the nonresolved emission band. ${ }^{27,28}$

The exact nature of this CTS defect was identified by quantum-chemical calculations in model systems containing fluorene groups and one fluorenone unit in the center $(\mathrm{FL})_{n}-$ FLO- $(\mathrm{FL})_{n}$. The energy of the CT state, which becomes the 

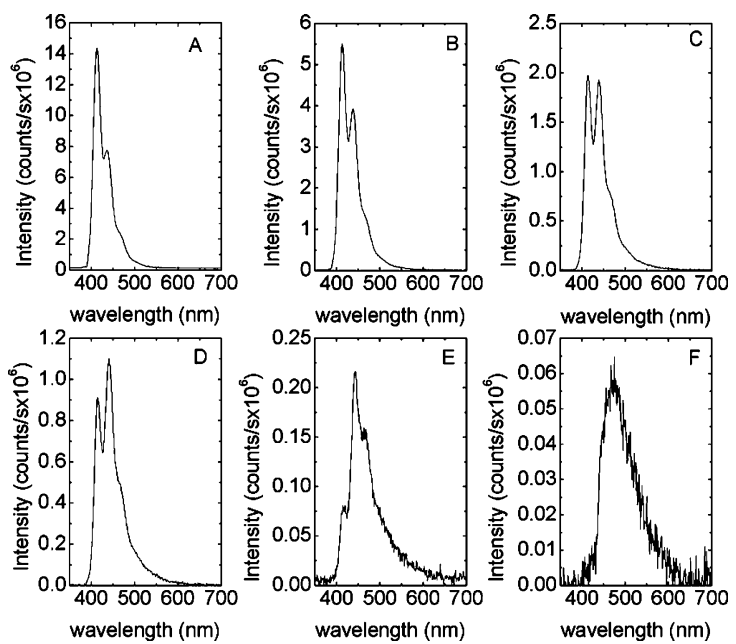

Figure 2. Emission spectra of $\mathrm{PF} 2 / 6$ in toluene solution as a function of 9FLO concentration: (A) 0 , (B) $1.4 \times 10^{-3}$, (C) $3.6 \times 10^{-3}$, (D) $6.2 \times 10^{-3}$, (E) $1.2 \times 10^{-2}$, and (F) $3.1 \times 10^{-2} \mathrm{~mol} / \mathrm{L}$. The decrease observed in the first vibrational reveals the reabsorption of PF2/6 emission by 9FLO At $3.1 \times 10^{-2} \mathrm{M}$, only the 9FLO emission is observed.

lowest energy state upon excitation, decreases by $0.32 \mathrm{eV}$ when going from FLO to the three-unit system FL-FLO-FL and then remains constant upon further increasing the chain length, ${ }^{29}$ showing that the system (FL-FLO-FL) is the appropriate model compound for our "CTS defect".

In some cases, solutions of polymers having random sections of chromophores with lower energy gaps have been shown to give dual photoluminescence spectra, with the higher energy band associated with the higher energy gap of the polymer and a red-shifted band corresponding to the lower energy guest. ${ }^{30}$ This is not normally the case in solutions of alternating copolymers in solution or films, where single emission band spectra are generally observed. Efficient energy transfer promoted by close proximity between the two polymer moieties causes quenching of the emission from higher energy sites. ${ }^{30}$

The observation in $\mathrm{PF} / \mathrm{FL}_{x}$ copolymers of a dual fluorescence shows that the energy transfer process between PF and CTS defects is not totally efficient. There are domains of the copolymer chain where the energy transfer channel does not compete with the intrinsic PF deactivation, possibly due to spatially self-localization of the PF excitations on structural defects $^{30}$ or due to energy funneling in the conformational disordered ensemble of conjugated segments., ${ }^{9,30}$

B. Intermolecular Effects of 9-Fluorenone on the Fluorescence Emission of PF2/6. In Figure 2, the emission spectra of PF2/6 $\left(10^{-3} \mathrm{mg} / \mathrm{mL}\right)$ mixed with 9-fluorenone (9FLO) in toluene solution are shown as a function of the 9-fluorenone concentration.

Increasing the 9-fluorenone concentration up to $3.1 \times 10^{-2}$ $\mathrm{mol} / \mathrm{L}$ leads to a decrease in the PF2/6 emission, with the effect being most pronounced on the first vibronic band, where the overlap between the PF2/6 emission and 9FLO absorption is largest (see Figure 1). Even at an extremely high 9FLO concentration $\left(3.1 \times 10^{-2} \mathrm{~mol} / \mathrm{L}\right)$, no CTS emission is observed, and only the weak emission band from 9FLO appears.

Results from fluorescence decays in toluene solution of pure PF2/6 and of PF2/6 mixed with 9FLO $\left(6.2 \times 10^{-3} \mathrm{~mol} / \mathrm{L}\right)$ are shown in Table 1 . Analysis of both decays collected at $448 \mathrm{~nm}$ shows an excellent fit to the sum of two exponential functions, with 20 and $360 \mathrm{ps}$, for the pure solution of PF2/6, and with sums of three exponentials, with 20 ps, 300 ps and 2 ns, when PF2/6 is mixed with 9FLO. Decay times around 40 and 370 ps
Table 1. Results from Fluorescence Decay Analysis of PF2/6 in Toluene Solution for Two Different 9FLO Concentrations

\begin{tabular}{lcccll}
\hline$[9 \mathrm{FLO}](\mathrm{mol} / \mathrm{L})$ & $\lambda_{\mathrm{em}}(\mathrm{nm})$ & $\tau_{3}(\mathrm{ps})$ & $\tau_{2}(\mathrm{ps})$ & $\tau_{1}(\mathrm{~ns})$ & $\chi^{2}$ \\
\hline 0 & & 20 & 360 & & \\
\multirow{2}{*}{$6.2 \times 10^{-3}$} & 448 & -0.451 & 1.000 & & 1.12 \\
& 448 & -0.323 & 0.988 & 0.012 & 0.99
\end{tabular}

were reported previously ${ }^{16}$ for PF2/6 in methylcyclohexane solution, with the faster component assigned to a conformational rearrangement of the lowest excited state, after energy migration from upper energy levels, and the slower one to the polyfluorene lifetime. The $2.0 \mathrm{~ns}$ component is in good agreement with the value reported before for 9FLO in toluene solution. ${ }^{20,31}$ The lack of acceptor emission following intermolecular quenching of PF emission by 9FLO clearly shows that through-space radiationless energy transfer between fluorene units and 9-fluorenone does not occur. Figure 2 strongly suggests that only the trivial reabsorption of PF emission by 9FLO is involved. This picture is confirmed by the time-resolved fluorescence decays (see Table 1). The observed reduction of the polyfluorene lifetime, from 360 to $300 \mathrm{ps,}$, could be artificial, resulting from the presence of the long 9FLO lifetime ( $2 \mathrm{~ns})$, due to direct excitation of 9FLO, in the single-wavelength three-exponential fit. However, even if that is not the case, a simple and qualitative calculation shows that intermolecular energy transfer between PF2/6 and 9FLO cannot be efficient under these conditions. RET would be efficient if $k_{\mathrm{Q}}[\mathrm{Q}] \gg \tau_{0}{ }^{-1}$, where $k_{\mathrm{Q}}$ is the quenching rate constant, [Q] is the quencher (9FLO) concentration, and $\tau_{0}$ is the polyfluorene lifetime. The criterion is not fulfilled at least for concentrations up to $6.2 \times 10^{-3} \mathrm{~mol} / \mathrm{L}, k_{\mathrm{Q}}[\mathrm{Q}]=5.5 \times 10^{-4}$ $\mathrm{ps}^{-1}$, and $\tau_{0}^{-1}=2.7 \times 10^{-3} \mathrm{ps}^{-1}$.

The short lifetime of the polyfluorene fluorescence, $360 \mathrm{ps}$, strongly suggests that dynamic intermolecular interactions are inhibited. For small molecules in nonviscous solvents, the most favorable case for a diffusion-controlled quenching process, the diffusion coefficient $D$ is approximately $5 \times 10^{-5} \mathrm{~cm}^{2} \mathrm{~s}^{-1}$; 14 if we consider the scenario for a molecule with a lifetime of 360 ps, the distance available for reaction would be around $2 \mathrm{~nm}$, slightly larger than the estimate of $1.6 \mathrm{~nm}$ for the Forster distance $R_{0}$ for PF2/6 and 9FLO. This simple calculation leads to a limiting distance above which no diffusion-controlled process can occur with singlet excited polyfluorene as a donor. This scaling factor is also important in the interpretation, based on the SANS results, of the possibility of interaction between well-separated units of the same chain, which are brought together by polymer chain motions within the polyfluorene lifetime.

C. Small-Angle Neutron Scattering (SANS) Studies of PF2/6 and PF/FL $x$ in Toluene Solution. Previous SANS studies of PF2/6 solutions ${ }^{32,33}$ indicate a wormlike conformation and a persistence length of $7 \mathrm{~nm}$, which is above the limiting encounter distance qualitatively calculated above in section B. Therefore, the possibility of the presence of rigid-rod (or hairyrod) segments larger than $2 \mathrm{~nm}$ in these copolymers has also been studied using SANS. Unfortunately, even with the high flux neutron source used, it is not possible to study polyfluorene solutions over the same low concentration $(\mu \mathrm{g} / \mathrm{mL})$ region used for the photophysical measurements, but higher concentration samples $(\mathrm{mg} / \mathrm{mL})$ are experimentally more appropriate for the SANS measurements. However, it has been suggested that there is no significant solvent effect or sign of interaction between the individual PF2/6 chains within the concentration regime $1-11 \mathrm{mg} / \mathrm{mL}{ }^{32,33}$ Furthermore, the classic coil formation of flexible polymers in solutions takes place at much higher 

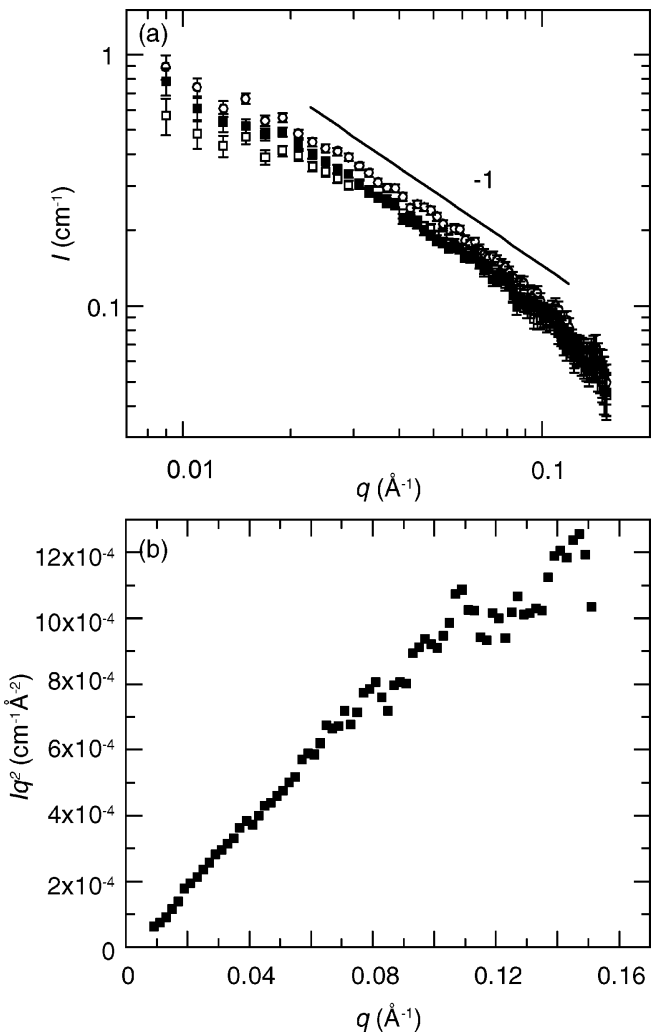

Figure 3. (a) SANS data of copolymers in deuterated toluene: $\mathrm{PF} /$ $\mathrm{FL}_{0.01}$ (open spheres), $\mathrm{PF} / \mathrm{FL}_{0.1}$ (open squares), and $\mathrm{PF} / \mathrm{FL}_{0.25}$ (solid squares). Solid line is the linear fit of the data of $\mathrm{PF} / \mathrm{FL}_{0.25}$ (lifted for clarity). The slope is $0.98 \pm 0.02$. (b) Kratky plot for $\mathrm{PF} / \mathrm{FL}_{0.25}$. The polymer concentration was $10 \mathrm{mg} / \mathrm{mL}$ and temperature $25^{\circ} \mathrm{C}$.

concentrations, ${ }^{34}$ and it is unlikely that these uncharged, relatively rigid polymers will show any significant conformational change over the concentration range from that used for the photophysical measurements to that for the SANS studies. Even in the case of polyelectrolytes, poly( $p$-phenylene)-type polymers have been found to take rodlike conformation in solution regardless of concentration or strength of intermolecular interaction. ${ }^{35}$ Therefore, if the copolymers are predominantly rigid in the high concentration regime, we assume that this is also the case for dilute solutions.

In Figure 3a, plots of SANS data of $\mathrm{PF} / \mathrm{FL}_{0.01}, \mathrm{PF} / \mathrm{FL}_{0.10}$, and $\mathrm{PF} / \mathrm{FL}_{0.25}$ solutions are given in $\log -\log$ representations. As the highest fluorenone fraction might result in the highest deviation from a stiff conformation, Figure $3 b$ shows a Kratky plot of the data of $\mathrm{PF} / \mathrm{FL}_{0.25}$ solution for comparison. Figure $3 \mathrm{a}$ shows a characteristic -1 decay in the middle of scattering curves, indicating that copolymers are mostly stiff in the size range of the observation window, at distances roughly from 4 to $70 \mathrm{~nm}$. It is noteworthy that all polymers show essentially similar characteristics although there is 25-fold difference in fluorenone fraction. Deviations from the linear shape of the curves are visible at higher $q$ in Figure $3 b$, indicating local deviations from the stiff conformation. This is attributed to bending of the chains at the level of a few monomer units. When this simple result is compared with the previous literature, ${ }^{32,33}$ it seems plausible that all the copolymers have an open wormlike conformation in toluene, behaving qualitatively in exactly the same way as $\mathrm{PF} 2 / 6$. We note that this characteristic is not always present for $\pi$-conjugated (semirigid) hairy-rod polymers and depends on the solvent. In particular, poly[2-methoxy-5-(2'-ethylhexyloxy)-1,4-phenylenevinylene] (MEH-PPV) has been observed to show -1 and -2 decays in chloroform and toluene solutions,

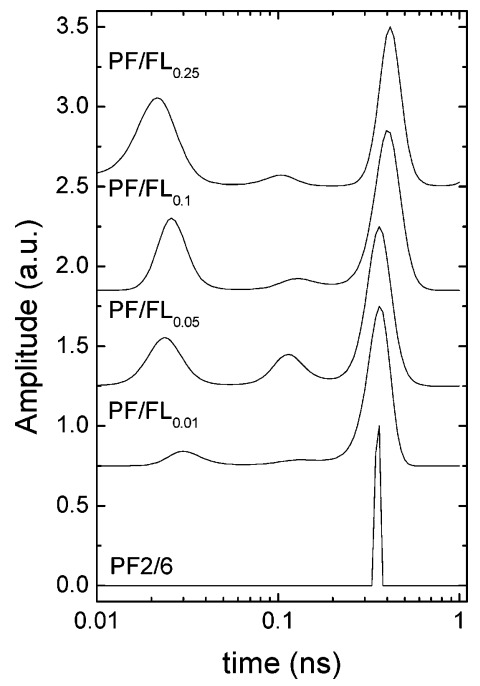

Figure 4. Maximum entropy method (MEM) analysis of PF2/6 and $\mathrm{PF} / \mathrm{FL}_{x}$ copolymers. For PF2/6 only a narrow distribution is observed at $360 \mathrm{ps}$. For $\mathrm{PF} / \mathrm{FL}_{x}$ copolymers, together with the distribution around $360 \mathrm{ps}$, two additional distributions are observed around 20 and 100 ps.

Table 2. Results $\left(\tau_{i}, A_{i}{ }^{415}, A_{i}{ }^{580}\right)$ Obtained from Global Analysis of Fluorescence Decays of PF2/6 and of PF/FL $\mathrm{F}_{x}$ in Toluene Solution at $295 \mathrm{~K}$

\begin{tabular}{|c|c|c|c|c|c|c|}
\hline compd & $\lambda_{\mathrm{em}}(\mathrm{nm})$ & & $\tau_{4}(\mathrm{ps})$ & $\tau_{3}(\mathrm{ps})$ & $\tau_{2}(\mathrm{ps})$ & $\tau_{1}(\mathrm{~ns})$ \\
\hline \multirow[t]{3}{*}{$\mathrm{PF} 2 / 6$} & & $\tau_{i}$ & 20 & 360 & & \\
\hline & 400 & $A_{i}^{400}$ & 0.4 & 0.6 & & \\
\hline & 415 & $A_{i}^{415}$ & -0.06 & 1.00 & & \\
\hline \multirow{3}{*}{$\mathrm{PF} / \mathrm{FL}_{0.01}$} & & $\tau_{i}$ & 31 & 203 & 389 & 6.49 \\
\hline & 415 & $A_{i}^{415}$ & 0.15 & 0.17 & 0.68 & 0 \\
\hline & 580 & $A_{i}^{580}$ & -0.14 & -0.08 & 0.34 & 0.66 \\
\hline \multirow[t]{3}{*}{$\mathrm{PF} / \mathrm{FL}_{0.05}$} & & $\tau_{i}$ & 22 & 128 & 372 & 6.55 \\
\hline & 415 & $A_{i}^{415}$ & 0.28 & 0.21 & 0.51 & 0 \\
\hline & 580 & $A_{i}^{580}$ & -0.15 & -0.1 & 0.11 & 0.89 \\
\hline \multirow{3}{*}{$\mathrm{PF} / \mathrm{FL}_{0.1}$} & & $\tau_{i}$ & 13 & 89 & 360 & 6.18 \\
\hline & 415 & $A_{i}^{415}$ & 0.43 & 0.14 & 0.43 & 0 \\
\hline & 580 & $A_{i}^{580}$ & -0.07 & -0.04 & 0.06 & 0.94 \\
\hline \multirow[t]{3}{*}{$\mathrm{PF} / \mathrm{FL}_{0.25}$} & & $\tau_{i}$ & 10 & 78 & 399 & 6.5 \\
\hline & 415 & $A_{i}^{415}$ & 0.57 & 0.12 & 0.31 & 0 \\
\hline & 580 & $A_{i}^{580}$ & -0.12 & -0.02 & 0.07 & 0.93 \\
\hline
\end{tabular}

respectively, the latter observation indicating disklike aggregates in which rod segments are laterally packed. ${ }^{36}$

D. Time-Resolved Fluorescence Decays of $\mathbf{P F} / \mathbf{F L}_{x}$ in Toluene Solution. Time-resolved fluorescence decays collected at $415 \mathrm{~nm}$ (polyfluorene emission) and $580 \mathrm{~nm}$ (charge transfer state emission) were performed at room temperature, with excitation at $390 \mathrm{~nm}$, for the homopolymer PF2/6 and for copolymers $\left(\mathrm{PF} / \mathrm{FL}_{x}\right)$ with fluorenone fraction ranging from $1 \%$ to $25 \%$.

Figure 4 shows the MEM analysis of fluorescence decays collected at $415 \mathrm{~nm}$ for PF2/6 and the PF/FL $\mathrm{FL}_{x}$ copolymers. For $\mathrm{PF} 2 / 6$, only a narrow decay time distribution around $361 \mathrm{ps}$ is found, which is in excellent agreement with the results from global analysis shown in Table 2, considering that the MEM software package used in this work cannot handle components appearing as a rise time (negative amplitudes).

In contrast to what happens with the homopolymer (PF2/6), three different time distributions, centered approximately on 400, 100 , and $25 \mathrm{ps}$, are found for the copolymers $\left(\mathrm{PF} / \mathrm{FL}_{x}\right)$. Analysis of Figure 4 clearly identifies the slower component with the homopolymer decay time and the two faster components as being due to the presence of fluorenone defects.

The MEM results show that sums of four exponential functions are necessary to describe the global analysis of PF/ 


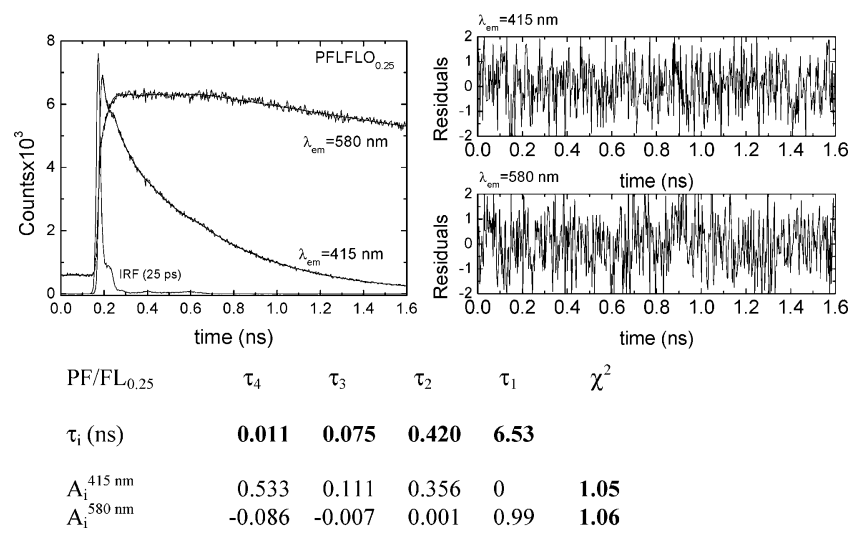

Figure 5. Fluorescence decay of $\mathrm{PF} / \mathrm{Fl}_{0.25}$ collected at 415 and 580 $\mathrm{nm}$ at $293 \mathrm{~K}$ and results from global analysis with decay times $\left(\tau_{i}\right)$, amplitudes $\left(A_{i}^{415}, A_{i}^{580}\right)$, and fitting parameters, weighted residuals, and $\chi^{2}$ values.

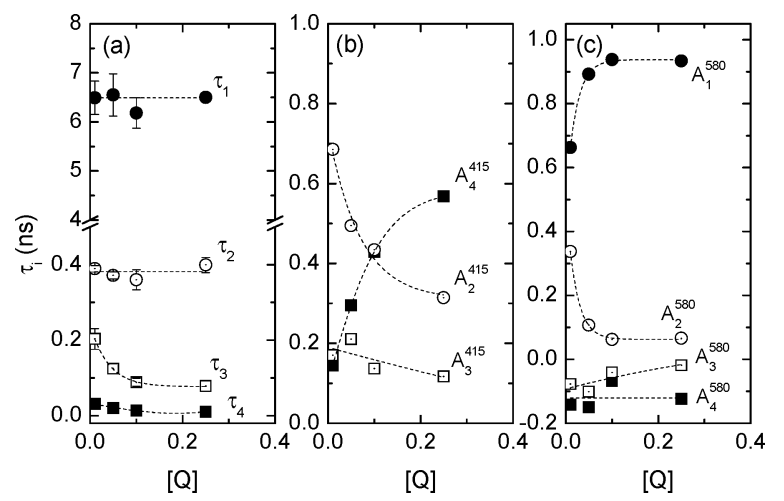

Figure 6. Global fit parameters of the fluorescence decays of $\mathrm{PF} / \mathrm{Fl}_{x}$ in toluene as a function of fluorenone fraction: (a) decay times $\tau_{i}$, (b) preexponential amplitudes at $415 \mathrm{~nm} A_{i},{ }^{415}$ (c) preexponential amplitudes

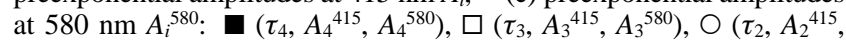
$\left.A_{2}{ }^{580}\right)$, and $\bullet\left(\tau_{1}, A_{1}{ }^{580}\right)$. Lines are merely guide lines.

$\mathrm{FL}_{x}$ fluorescence decays, with three decay components to fit the decay collected at $415 \mathrm{~nm}, \mathrm{PF}$ emission, and one more component, linked to the CTS emission at $580 \mathrm{~nm}$.

The global analysis of the fluorescence decay of $\mathrm{PF} / \mathrm{FL}_{0.25}$, collected at 415 and $580 \mathrm{~nm}$, is shown in Figure 5. Again, three decay components are identified at $415 \mathrm{~nm}, 11,75$, and 420 ps, in good agreement with the MEM analysis; a fourth component of $6.5 \mathrm{~ns}$ is also detected when the emission is collected at $580 \mathrm{~nm}$.

Results, average decay times and preexponential amplitudes, obtained from the global analysis of at least four fluorescence decays for each $\mathrm{PF} / \mathrm{FL}_{x}$ copolymer in toluene solution, are presented in Figure 6 as a function of fluorenone fraction. Table 2 summarizes the results obtained from global analysis of fluorescence decays for all the copolymers PF/FL $x$ and also for the decay obtained with the homopolymer PF2/6, collected at the emission onset (400 $\mathrm{nm})$ and maximum (415 nm).

Four decay components are identified for all the copolymers. The slower component, around $6.5 \mathrm{~ns}$, is only important at 580 $\mathrm{nm}$ and is clearly identified with the CTS lifetime. The intermediate component, varying from 360 to 400 ps, appears as a decay time at both wavelengths and is more important at $415 \mathrm{~nm}$ than at $580 \mathrm{~nm}$. This component is clearly identified with the polyfluorene lifetime. The two faster components, varying from 10 to 30 ps and from 78 to 203 ps, appear as decay times at $415 \mathrm{~nm}, \mathrm{PF}$ emission, and as rise times at 580 nm, CTS emission. These two components are clearly connected with the CTS excited-state population at the expense of the $\mathrm{PF} 2 / 6$ excited state.

The slower component has previously been assigned ${ }^{20}$ to singlet polyfluorene excitations that migrate along the manifold of conjugated segments prior to meeting a CTS defect and suffering quenching. The presence of a faster component, below the temporal resolution of our system, was strongly suggested to be identified with the presence of close PF-CTS defect pairs ${ }^{20}$ singlet excitations associated with these close PF-CTS pairs do not have to migrate to meet a CTS defect and suffer quenching.

\section{Data Analysis}

In summary, the use of discrete sums of exponential functions to describe the $\mathrm{PF} / \mathrm{FL}_{x}$ fluorescence kinetics suggests four different species: (i) CTS defects, which are the acceptors of energy transfer from the polyfluorene donor; (ii) slow-quenched polyfluorene singlet excitations ( $\left.\mathrm{PF}_{\mathrm{qs}} \mathrm{low}\right)$, polyfluorene excitations located far from CTS defects but free to move along the polymer chain by energy hopping and reach a quenching center; (iii) unquenched polyfluorene singlet excitations $\left(\mathrm{PF}_{\mathrm{unq}}\right)$; and (iv) fast-quenched polyfluorene singlet excitations $\left(\mathrm{PF}_{\mathrm{qfast}}\right)$, where downhill energy migration along the polymer backbone is strongly affected by conformational disorder that appears from bends and twists of the polymer backbone and that leads to spatial self-localization of singlet excitations. ${ }^{37,38}$ This last case may be quenched (if located close to a CTS defect) or not depending on the distance between them and the CTS defects.

In Scheme 2, the mechanism proposed to analyze the experimental results is described. $Q$ represents the fluorenone fraction; $k_{\text {unq }}$ and $k_{\mathrm{CTS}}$ represent respectively the rate constant for radiative decay of polyfluorene and CTS defects. The slower quenching process is described by $k_{1}(Q) 2(1-\beta)$. $k_{1}$ represents the observed quenching rate constant itself, the factor 2 accounts for the fact that each PF excitation can be quenched by two different acceptors located in opposite directions along the copolymer chain, and the factor $(1-\beta)$ accounts for the probability that the excitation occurs far from a defect. Finally, the fast quenching regime is described by $k_{2}(Q) \beta$, where $k_{2}$ is the rate constant itself and $\beta$ accounts for the probability that the excitation occurs close to a CTS defect.

The expressions $\alpha(1-\beta) I_{0},(1-\alpha)(1-\beta) I_{0}$, and $\beta I_{0}$ represent the fraction of light absorbed by the different $\mathrm{PF}$ species, respectively $P F_{\text {unq }}, P F_{\mathrm{q}-\text { slow }}$, and $P F_{\mathrm{q}-\text { fast }}$, where $I_{0}$ represents the fraction of light absorbed by all PF species at the excitation wavelength. Direct light absorption by CTS defects is denoted by $\left(1-I_{0}\right)$.

The time evolution of the excited-state concentration of the four kinetic species in Scheme 2 is described by eq 1:

$$
\begin{aligned}
& \frac{\mathrm{d}}{\mathrm{d} t}\left[\begin{array}{l}
P F_{\mathrm{unq}}^{*} \\
P F_{\mathrm{q}-\text { slow }}^{*} \\
P F_{\mathrm{q}-\text { fast }}^{*} \\
C T S^{*}
\end{array}\right]= \\
& {\left[\begin{array}{llll}
-k_{\text {unq }} & 0 & 0 & 0 \\
0 & -\left(k_{1}(Q) 2(1-\beta)+k_{\text {unq }}\right) & 0 & 0 \\
0 & 0 & -\left(k_{2}(Q) \beta+k_{\text {unq }}\right) & 0 \\
0 & k_{1}(Q) 2(1-\beta) & k_{2}(Q) \beta & -k_{\mathrm{CTS}}
\end{array}\right]\left[\begin{array}{l}
P F_{\text {unq }}^{*} \\
P F_{\mathrm{q}-\text {-slow }}^{*} \\
P F_{\mathrm{q}-\text { fast }}^{*} \\
C T S^{*}
\end{array}\right]}
\end{aligned}
$$

The integration of the differential equation (1) describing the time evolution of the excited-state concentration of the four species (see ref 20 for more details) leads to the expressions 
Scheme 2

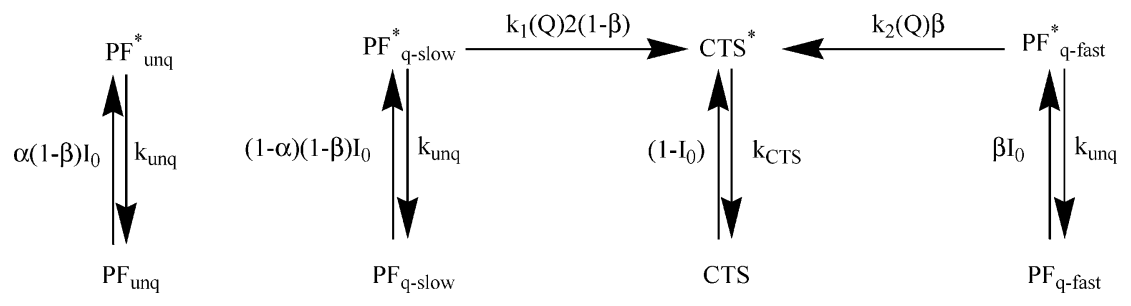

(2-4) describing the temporal evolution of the PF species in the excited state:

$$
\begin{gathered}
{\left[P F_{\text {unq }}^{*}\right](t)=\alpha(1-\beta) \mathrm{e}^{-t k_{\text {unq }}}} \\
{\left[P F_{\mathrm{q}-\text { slow }}^{*}\right](t)=(1-\alpha)(1-\beta) \mathrm{e}^{-t\left(k_{1}(Q) 2(1-\beta)+k_{\text {unq }}\right)}} \\
{\left[P F_{\mathrm{q}-\text { fast }}^{*}\right](t)=\beta \mathrm{e}^{-t\left(k_{2}(Q) \beta+k_{\text {unq }}\right)}}
\end{gathered}
$$

The relations between the kinetic parameters in Scheme 2 and the experimental observables decay times $\left(\tau_{i}\right)$ and preexponential amplitudes $\left(A_{i}^{415}\right)$, are given by eqs $5-10$.

$$
\begin{gathered}
\tau_{1}{ }^{-1}=k_{\mathrm{CTS}} \\
\tau_{2}{ }^{-1}=k_{\text {unq }} \\
\tau^{-1}=\tau_{2}^{-1}+k_{1}(Q) 2(1-\beta) \\
\tau_{4}^{-1}=\tau_{2}^{-1}+k_{2}(Q) \beta \\
A_{2}^{415}+A_{3}^{415}+A_{4}^{415} \\
\alpha=\frac{A_{2}^{415}}{A_{2}^{415}+A_{3}^{415}}
\end{gathered}
$$

The fractions of the kinetically different polyfluorene units are represented in Figure 7 as a function of the fluorenone fraction. A qualitative analysis of the data shows that the fraction of closely spaced PF-CTS pairs $(\beta)$ increases as expected with the fluorenone fraction. The fraction of unquenched units $\alpha(1$ $-\beta$ ) decreases with the copolymer fluorenone content; obviously with more CTS defects it is more probable for any excitation to be quenched. Finally, the fraction of quenched PF units that are located far way from a CTS defect $(1-\alpha)(1-$ $\beta$ ) initially increases with the 9FLO fraction, but when the fast

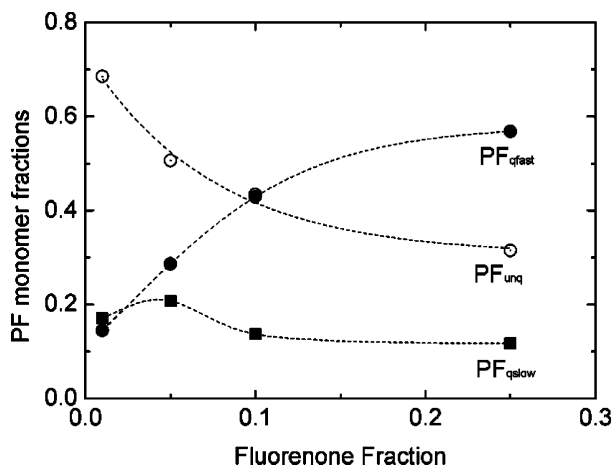

Figure 7. Dependence with $9 F L O$ content of $P F$ fractions, $P_{\text {unq }}$,

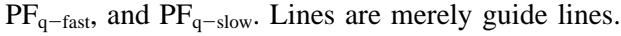

process starts to be more important, the contribution of this component decreases.

In Figure 8, the quenching rate constants for both slow $\left(k_{1}\right)$ and fast $\left(k_{2}\right)$ regimes, determined from eqs 7 and 8 rewritten respectively in the form $\left(\tau_{3}{ }^{-1}-\tau_{2}{ }^{-1}\right) /[2(1-\beta)]=k_{1}(Q)$ and $\left(\tau_{4}{ }^{-1}-\tau_{2}{ }^{-1}\right) / \beta=k_{2}(Q)$ are plotted against the fluorenone fraction.

The rate constant for the slower regime, $k_{1}$, is in excellent agreement with the previously reported ${ }^{20}$ value of $0.12 \mathrm{ps}^{-1}(1.2$ $\left.\times 10^{-11} \mathrm{~s}^{-1}\right)$. The slower quenching regime is dependent on the fluorenone fraction, such that with increasing $Q$ it becomes more probable that an excitation finds a CTS defect, analogous to what happens in a diffusive quenching process.

The determination of the quenching rate constant associated with the fast regime, $k_{2}$, is subject to a large experimental error $(30 \%)$. Note that $k_{2}$ is mainly controlled by the fast component, which is close to the temporal resolution of the TCSPC apparatus, and with low importance on the overall decay. However, and despite this large experimental error, $k_{2}$ can qualitatively be fitted with eq 11 , with $k_{0}=0.12 \mathrm{ps}^{-1}$ and $\alpha=$ 0.006 , where $k_{0}$ represents the maximum rate constant and $\alpha$ is a parameter without physical meaning, suggesting that the process is described by the electron exchange mechanism.

$$
k_{2}(Q)=k_{0} \mathrm{e}^{-\alpha / Q}
$$

In fact, increasing $Q$ would decrease the average minimum distance between a self-localized $P F_{\mathrm{q}-\text { fast }}$ excitation and a possible CTS defect, which suggests that eq 11 simply describes the qualitative dependence with distance for the electron exchange mechanism ${ }^{14} k_{\mathrm{ET}}=k_{0} \mathrm{e}^{-R}$, where $k_{0}$ is the maximum rate constant for energy transfer, occurring when donor and acceptor are at the collision distance $R_{0}$ and $R$ is the separation between donor and acceptor when they are further apart than $R_{0}$.

The fit is crude, in part because we only have a few experimental points in this region, but the fact that fitting $k_{2}$ with eq 11 leads to $k_{0}$ equal to $k_{1}$ suggests that what is in fact

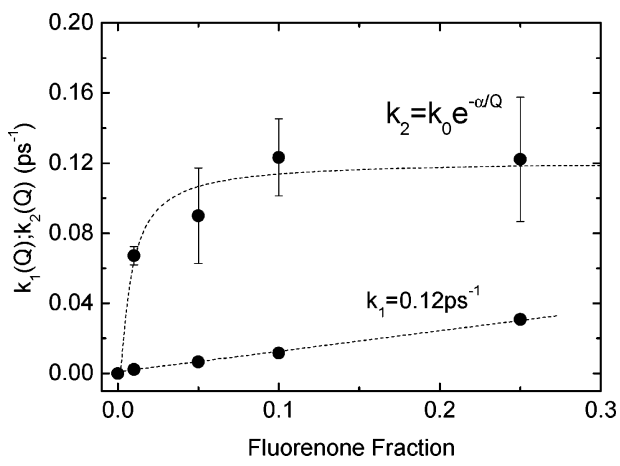

Figure 8. Determination of $k_{1}$ and $k_{2}$ using respectively eqs 7 and eq 8. The rate constant associated with the slower time regime shows a linear dependence with the 9FLO content. The fast time regime suggests an exponential dependence with 9FLO fraction, compatible with a shortrange mechanism. 
controlling the quenching process is not the energy migration along the chain but instead the energy transfer from PF units to CTS defects. This corrects our previous findings ${ }^{20}$ and establishes a lower limit ${ }^{39,40}$ for the magnitude of the energy migration rate constant in these systems of $0.12 \mathrm{ps}^{-1}\left(1.2 \times 10^{-11} \mathrm{~s}^{-1}\right)$. This value is in excellent agreement with recent reports ${ }^{7-9}$ where the intramolecular energy migrations rate constant is computed for oligoindenofluorenes to be in the range of $0.06-0.25 \mathrm{ps}^{-1}$.

Stretched Exponential Function in Decay Data Analysis. The description of fluorescence time decays by a sum of discrete exponentials has the advantage of providing a clear physical meaning for the fitting parameters, ${ }^{41}$ decay times, and preexponential amplitudes. Basically, the assumption of a fit with $N$ exponential functions establishes the idea that equal numbers of different kinetic species are present in the system. ${ }^{42}$ In the simplest cases the identification of such species is evident, but frequently that is not the case and this empirical approach has been questioned. .,43 $^{1}$

The lack of a unique kinetic scheme to rationalize the fitting parameters and the observation that a lifetime distribution can be successfully fitted by a sum of discrete exponential functions with two or three terms are the main points against the multiexponential approach. ${ }^{1,42,43}$

The problem can be in part, but not always, overcome, if narrow and well-separated time distributions can be identified in MEM plots and if data obtained from other techniques can be used as a functional block to test the model predictions.

Alternatively, the use of a stretched exponential function (eq 12) has been frequently proposed ${ }^{1,41}$ to describe the timedependent donor emission in photon-harvesting polymer systems. In this equation, $\tau_{0}$ is the donor lifetime in the absence of energy acceptors, $A$ is a parameter proportional to the local acceptor concentration, and $0<\beta \leq 1$ is an empirical parameter directly connected with the physical dimensions where the fluorophores are distributed. ${ }^{1}$

$$
I(t)=\mathrm{e}^{\left(-t / \tau_{0}\right)-A\left(t / \tau_{0}\right)^{\beta}}
$$

Figure 9 shows the analysis of $\mathrm{PF} / \mathrm{FL}_{0.25}$ fluorescence decay collected at $415 \mathrm{~nm}$, with (a) a stretched exponential and (b) a sum of three discrete exponential functions. The fit with eq 12 is rather poor when compared with the results from a sum of three discrete exponential functions. The same observation can be made for all the copolymers. Table 3 summarizes the resulting fit parameters $\left(\tau_{0}, A, \beta\right)$, and $\chi^{2}$ values, obtained from deconvolution with a stretched exponential (eq 12), for PF2/6 fluorescence decays collected at 400 and $415 \mathrm{~nm}$ and for PF/ $\mathrm{FL}_{x}$ fluorescence decays collected at $415 \mathrm{~nm}$.

Despite the quality of the fit, interpretation of results obtained from the decay analysis with eq 12 is shown to be in agreement with the interpretation obtained from global analysis with a sum of discrete exponential functions. The PF2/6 fluorescence decay collected at $400 \mathrm{~nm}$, the polyfluorene emission onset, is characterized by large $A$ and small $\beta$ values, while the decay collected at $415 \mathrm{~nm}$ shows a small $A$ value and $\beta$ equal to 1 . The emission collected at $400 \mathrm{~nm}$ clearly results from an nonrelaxed state, ${ }^{16}$ the obtained high $A$ value is compatible with a higher "local concentration of acceptors" (the relaxed state), and the highly nonexponential behavior $(\operatorname{small} \beta$ ) denotes the coupling to the relaxed state, which emits at $415 \mathrm{~nm}$ with a small $A$ value, low local concentration of acceptors, and an exponential decay law $(\beta=1)$.

Contrary to what happens with $\mathrm{PF} 2 / 6$, fluorescence decays of $\mathrm{PF} / \mathrm{FL}_{x}$ collected at $415 \mathrm{~nm}$ can no longer be described by a single-exponential law $(\beta=1)$. Increasing $A$ and decreasing $\beta$
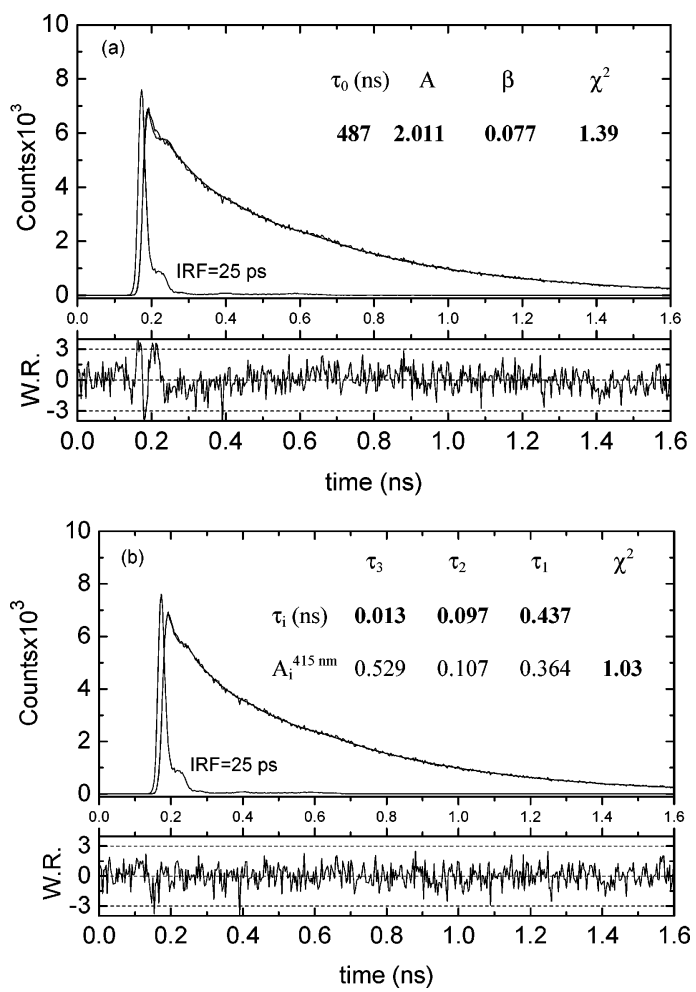

Figure 9. Fluorescence decay of $\mathrm{PF} / \mathrm{Fl}_{0.25}$ collected at $415 \mathrm{~nm}$ at 293 K. Results obtained from (a) analysis with a stretched exponential $\left(\tau_{0}\right.$ $A, \beta$ ) (see eq 12) and (b) analysis with a sum of three exponential functions, decay times $\left(\tau_{i}\right)$, and amplitudes $\left(A_{i}^{415}\right)$. The fitting parameters, weighted residuals, and $\chi^{2}$ values are also shown for each analysis.

Table 3. Results, Fit Parameters $\left(\tau_{0}, \mathbf{A}, \boldsymbol{\beta}\right)$, and $\chi^{2}$ Values, Obtained from Deconvolution with a Stretched Exponential, of Fluorescence Decays of PF2/6 and of PF/FL $x$ in Toluene Solution at $295 \mathrm{~K}$

\begin{tabular}{lccccc}
\hline compd & $\lambda_{\text {em }}(\mathrm{nm})$ & $\tau_{0}(\mathrm{~ns})$ & \multicolumn{1}{c}{$A$} & \multicolumn{1}{c}{$\beta$} & $\chi^{2}$ \\
\hline PF2/6 & 400 & 0.367 & 1.402 & 0.053 & 1.45 \\
& 415 & 0.367 & 0.048 & 1.0 & 1.16 \\
PF/FL $_{0.01}$ & 415 & 0.419 & 0.324 & 0.415 & 1.58 \\
PF/FL $_{0.05}$ & 415 & 0.433 & 0.801 & 0.364 & 1.1 \\
PF/FL $_{0.1}$ & 415 & 0.441 & 1.379 & 0.108 & 1.12 \\
PF/FL $_{0.25}$ & 415 & 0.487 & 2.011 & 0.077 & 1.39
\end{tabular}

values are obtained with increasing fluorenone fraction. At higher fluorenone fractions the local concentration of acceptors increases, giving larger $A$ values; the importance of a fast component in the overall decay will also increase with fluorenone fraction, leading to small $\beta$ values. Apart from the identification of different physical kinetic species, this picture agrees with the description obtained when a sum of discrete exponential functions is used. However, the physical meaning of parameters $\beta$ and $\tau_{0}$ is not clear.

The PF2/6 lifetime determined either from decay analysis with a sum of exponentials or with eq 12 is about 360 ps, MEM and global analysis with a sum of exponentials return values (see Figure 4 and $\tau_{2}$ values in Table 2), in good agreement with the polyfluorene lifetime. However, when eq 12 is used to fit the fluorescence decays of PF/FL ${ }_{x}$ copolymers, the lifetime of PF2/6 is never recovered. In fact, $\tau_{0}$ increases with fluorenone fraction, assuming a value of $487 \mathrm{ps}$ for $\mathrm{PF} / \mathrm{FL}_{0.25}$, about $120 \mathrm{ps}$ longer than the polyfluorene lifetime. Also, the physical meaning of $\beta$ is hard to explain; this parameter is supposed to reflect the physical dimensions and the type of deactivation processes involved. For a quenching process controlled by diffusion contact $\beta=0.5$ and for a single-step RET by the dipole-dipole mechanism $\beta$ will be $0.16,0.33$, and 0.5 respectively for one, two, or three dimensions. ${ }^{41}$ Fluorescence decays in fractals are 
also described by eq 12, 1-dimensional systems with donor decaying only by a random walk to a trapping site will give $\beta$ $\in[0.5,1],{ }^{1,45}$ and if a single-step energy transfer is also involved $\beta<0.5$ can also be obtained. ${ }^{1,45}$

In our case, from the SANS measurements geometry changes were not detected in the copolymer chain upon increasing the fluorenone fraction, but $\beta$ still shows a strong dependence with 9FLO content with values always below 0.5 . We could speculate that a mix of one-dimensional random walk with single-step energy transfer is present, ${ }^{45}$ which leads to a conclusion similar to that obtained from global and MEM analysis. However, in the absence of a proper theoretical model describing the energy relaxation in these systems with eq 12 , it is more prudent to assume that $A, \beta$, and $\tau_{0}$ more strongly reflect the fractional weight of the three components detected by MEM and global analysis than to resort to a particular physical meaning of these properties, with the disadvantage that no direct relation exists between the fitting parameters and decay times.

Time-Resolved vs Steady-State Data: The Stern-Volmer Plot. Time-resolved and steady-state data can be compared without the assumption of any particular kinetic mechanism. ${ }^{1}$ The PF steady-state intensity $I^{S S}$ at $415 \mathrm{~nm}$ can be obtained from fluorescence decays collected at $415 \mathrm{~nm}$ using eq 13 .

$$
I^{S S}=\int_{0}^{\infty} I(t) \mathrm{d} t=\sum_{i}^{N} A_{i} \tau_{i}
$$

When used in the form of a Stern-Volmer plot, either steadystate or time-resolved data should give the same dependence upon the quencher concentration. However, in contrast with time-resolved data, the Stern-Volmer plots obtained from steady-state data frequently show an upward curvature. , $^{1,44}$ The presence of static quenching or an ultrafast component, below the instrument temporal resolution, is normally suggested to explain the observed discrepancy.

In the case of $\mathrm{PF} / \mathrm{FL}_{x}$ copolymers, despite the observation of an upward curvature in the Stern-Volmer plot, no spectral evidence for static quenching was found (Figure 1). The presence of an undetectable faster component, below the temporal resolution used, was then suggested to explain this behavior. ${ }^{20}$ However, in the present study, despite the fact that the faster component has now been resolved, the discrepancy between steady state and time resolved is still observed at high fluorenone fractions. The reason for this is still unclear. However, besides in addition to trivial explanations, such as the presence of reabsorption of PF emission by CTS defects, which we have tested and ruled out, there are other situations that can explain this observation. ${ }^{1}$ Changes in the fluorescence quantum yield of PF after covalent attachment of 9FLO units and the existence of an initially PF excited state that can sensitize the CTS acceptor before relaxation to the PF singlet emissive state are among the possible causes. This is not entirely surprising given the complexity of initial excitation delocalization observed in other conjugated polymers. ${ }^{47}$ Despite the merely suggestive presence of a precursor state, the observation of a larger positive sum for the preexponential amplitudes at 580 $\mathrm{nm}$, the CTS emission, gives some additional support for this explanation.

\section{Conclusions}

The luminescence kinetics in $\mathrm{PF} / \mathrm{FL}_{x}$ copolymers was studied in dilute toluene solution. SANS results show a predominant 1-dimensional wormlike conformation for all the copolymers studied. In photophysical studies, two different time regimes were identified in the population of intramolecular induced fluorenone defects. The slower time regime shows a linear dependence on the fluorenone fraction, compatible with excitation incoherent hopping along the polymer backbone. The fast time regime suggests an exponential dependence with $Q^{-1}$, compatible with a short range (Dexter) mechanism. The rate constant determined from the slower time regime $\left(1.2 \times 10^{-11}\right.$ $\mathrm{s}^{-1}$ ) matches the maximum rate constant for the fast process, suggesting that what is in fact controlling the quenching process is the energy transfer from PF units to induced defects.

Acknowledgment. The authors are grateful to Scandiucci de Freitas and Ulli Scherf for supplying the copolymers and to George Striker for making his deconvolution program available. The SANS experiments have been supported by the European Commission under the 6th Framework Programme through the Key Action: Strengthening the European Research Area, Research Infrastructures, Contract No. HII3-CT-2003-505925. We thank also Steve King of the Rutherford Appleton Laboratory for assistance and discussions with these measurements. This work was funded by One North-East via the County Durham Sub-regional Partnership, project SP/082, and the nanotechnology UIC project. H.D.B. is also grateful to POCTI/ FCT/FEDER for further financial support.

\section{References and Notes}

(1) Webber, S. E. Chem. Rev. 1990, 90, 1469-1482.

(2) Herz, L. M.; Silva, C.; Grimsdale, A. C.; Mullen, K.; Phillips, R. T. Phys. Rev. B 2004, 70, 165207.

(3) Bodunov, E. N.; Berberan-Santos, M. N.; Martinho, J. M. G. Opt Spektrosk. 2001, 91, 740-749; Opt. Spectrosc. 2001, 91, 694-703.

(4) Bodunov, E. N.; Berberan-Santos, M. N.; Martinho, J. M. G. Chem Phys. Lett. 2001, 340, 137-141.

(5) Sokolov, I. M.; Mai, J.; Blumen, A. J. Lumin. 1998, 76\&77, 377380.

(6) Arkhipov, V. L.; Emelianova, E. V.; Bassler, H. Phys. Rev. B 2004, $70,205$.

(7) Beljonne, D.; Pourtois, G.; Shuai, Z.; Hennebicq, E.; Scholes, G. D.; Brédas, J. L. Synth. Met. 2003, 137, 1369-1371.

(8) Brédas, J. L.; Beljonne, D.; Coropceanu, V.; Cornil, J. Chem. Rev. 2004, 104, 4971-5003.

(9) Hennebicq, E.; Pourtois, G.; Scholes, G. D.; Herz, L. M.; Russel, D. M.; Silva, C.; Setayesh, S.; Grimsdale, A.; Mullen, K.; Brédas, J. L.; Beljonne, D. J. Am. Chem. Soc. 2005, 127, 4744-4762.

(10) Akcelrud, L. Prog. Polym. Sci. 2003, 28, 875-962.

(11) Brabec, C. J.; Sariciftci, N. S.; Hummelen, J. C. Adv. Funct. Mater. 2001, 11, 15-26.

(12) Ho, H. A.; Doré, K.; Boissinot, M.; Bergeron, M. G.; Tanguy, R. M.; Boudreau, D.; Leclerc, M. J. Am. Chem. Soc. 2005, 127, 1267312676.

(13) Westenhoff, S.; Daniel, C.; Friend, R. H.; Silva, C.; Sundstrom, V.; Yartsev, A. J. Chem. Phys. 2005, 122, 94903.

(14) Turro, N. J. In Modern Molecular Photochemistry; Benjamim/ Cummings: Menlo Park, CA, 1978.

(15) Ruseckas, A.; Namdas, E. B.; Theander, M.; Svensson, M.; Yartsev A.; Zigmantas, D.; Andersson, M. R.; Inganas, O.; Sundstrom, V. J. Photochem. Photobiol. A 2001, 144, 3-12.

(16) Dias, F. B.; Maçanita, A. L.; Melo, J. S.; Burrows, H. D.; Guntner, R.; Scherf, U.; Monkman, A. P. J. Chem. Phys. 2003, 118, 71197126.

(17) Grage, M. M. L.; Pullerits, T.; Ruseckas, A.; Theander, M.; Inganas, O.; Sundstrom, V. Chem. Phys. Lett. 2001, 339, 96-102.

(18) Scholes, G. D.; Larsen, D. S.; Fleming, G. R.; Rumbles, G.; Burn, P L. Phys. Rev. B 2000, 61, 13670-13678.

(19) Sluch, M. I.; Godt, A.; Bunz, U. H. F.; Berg, M. A. J. Am. Chem. Soc. 2001, 123, 6447-6448.

(20) Dias, F. B.; Maiti, M.; Hintschich, S. I.; Monkman, A. P. J. Chem. Phys. 2005, 122, 54904.

(21) Romaner, L.; Piok, T.; Gadermaier, C.; Guentner, R.; Scandiucci de Freitas, P.; Scherf, U.; Cerullo, G.; Lanzani, G.; List, E. J. W. Synth. Met. 2003, 139, 851-854.

(22) de Freitas, P. S.; Scherf, U.; Collon, M.; List, E. J. W. e-Polym. 2002, art. no. 009.

(23) Striker, G. In Deconvolution and Reconvolution of Analytical Signals; DPIC: Nancy, 1982. 
(24) Beechem, J. M.; Gratton, E. Fluorescence Spectroscopy Data Analysis Environment A Second Generation Global Analysis Program. In Time Resolved Laser Spectroscopy in Biochemistry; Proc. SPIE 1988, 909, 70-81.

(25) Brochon, J.; Livesey, A. K.; Pouget, J.; Valeur, B. Chem. Phys. Lett. 1990, 174, 517-522.

(26) Heenan, R. K.; Penfold, J.; King, S. M. J. Appl. Crystallogr. 1997, 30, 1140-1147.

(27) Kulkarni, A. P.; Kong, X.; Jenekhe, S. A. J. Phys. Chem. B 2004 108, 8689-8701.

(28) Hintschich, S. I.; Rothe, C.; Sinha, S.; Monkman, A. P.; de Freitas, P. S.; Scherf, U. J. Chem. Phys. 2003, 119, 12017-12022.

(29) Zojer, E.; Pogantsch, A.; Hennebicq, E.; Beljonne, D.; Brédas, J. L.; de Freitas, P. S.; Scherf, U.; List, E. J. W. J. Chem. Phys. 2002, 117, 6794-6802.

(30) Morgado, J.; Charas, A.; Martinho, J. M. G.; Alcácer, L. Synth. Met. 2005, $154,81-84$

(31) Biczok, L.; Berces, T.; Marta, F. J. Phys. Chem. 1993, 97, 88958899

(32) Fytas, G.; Nothofer, H. G.; Scherf, U.; Vlassopoulos, D.; Meier, G Macromolecules 2002, 35, 481-488.

(33) Knaapila, M.; Stepanyan, R.; Torkkeli, M.; Lyons, B. P.; Ikonen, T. P.; Almásy, L.; Foreman, J. P.; Serimaa, R.; Güntner, R.; Scherf, U.; Monkman, A. P. Phys. Rev. E 2005, 71, 041802.

(34) Grosberg, A. Y.; Khokhlov, A. R. Statistical Physics of Macromolecules; American Institute of Physics: New York, 1994
(35) Guilleaume, B.; Blaul, J.; Wittemann, M.; Rehahn, M.; Ballauff, M. J. Phys.: Condens. Matter 2000, 12, A245-A251.

(36) Ou-Yang, W. C.; Chang, C. S.; Chen, H. L.; Tsao, C. S.; Peng, K. Y.; Chen, S. A.; Han, C. C. Phys. Rev. E 2005, 72, 031802.

(37) List, E.; Leising, G. Synth. Met. 2004, 141, 211-218.

(38) Kersting, R.; Mollay, B.; Rusch, M.; Wenisch, J.; Leising, G.; Kauffmann, K. F. J. Chem. Phys. 1997, 106, 2850-2864.

(39) Rauscher, U.; Bassler, H. Macromolecules 1990, 23, 398-405.

(40) Kenkre, V. M.; Parris, P. E.; Schmid, D. Phys. Rev. B 1985, 32, 49464955.

(41) Berberan-Santos, M. N.; Bodunov, E. N.; Valeur, B. Chem. Phys. 2005, $315,171-182$.

(42) Lakowicz, J. R. Principles of Fluorescence Spectroscopy, 2nd ed. Kluwer Academic/Plenum Publishers: New York, 1999; pp 129, 130.

(43) Siemiarczuk, A.; Ware, W. R. J. Phys. Chem. 1989, 93, 7609-7618.

(44) Nemzek, T. L.; Ware, W. R. J. Chem. Phys. 1975, 62, 477-489.

(45) Bai, F.; Chang, C.-H.; Webber, S. E. Macromolecules 1986, 19, 24842494.

(46) Zelent, B.; Kusba, J.; Gryczynski, I.; Johnson, M. L.; Lakowicz, J. R. Biophys. Chem. 1998, 73, 53-75.

(47) Ruseckas, A.; Wood, P.; Samuel, I. D.; Webster, G. R.; Mitchell, W. J.; Burn, P. L.; Sundstrom, V. Phys. Rev. B 2005, 72, 115214.

MA052505L 